\title{
GRADED PERMUTATION MODULES AND A THEOREM OF QUILLEN
}

\author{
ANER SHALEV
}

(Communicated by Maurice Auslander)

\begin{abstract}
A theorem of Quillen, which presents graded group algebras as certain enveloping algebras, is extended to permutation modules. In positive characteristic this provides a functorial version to Alperin's recent extension of Jennings's theory.
\end{abstract}

\section{INTRODUCTION}

Let $F$ be a fixed field of characteristic $p$ (which may be either zero or positive). For a group $G$, consider the group algebra $F G$, and let $\Delta$ denote its augmentation ideal. Define the graded group algebra $\operatorname{gr}(F G)$ by

$$
\operatorname{gr}(F G):=\bigoplus_{n \geq 0} \Delta^{n} / \Delta^{n+1}
$$

In 1968 Quillen described $\operatorname{gr}(F G)$ as an enveloping algebra of a certain Lie algebra [6]. In order to formulate his result, denote by $D_{n}$ the $n$th dimension subgroup of $G$ over $F$; namely,

$$
D_{n}:=G \cap\left(1+\Delta^{n}\right), \quad n \geq 1 .
$$

A group-theoretic description of these subgroups was given by Jennings and Lazard in [3-5] (see also [7]). In particular, it is known that $D_{n} / D_{n+1}$ is abelian; it is torsionfree if $p=0$ and has exponent $p$ otherwise. Set

$$
L(G):=\bigoplus_{n \geq 1} D_{n} / D_{n+1} .
$$

It is well known that $L(G)$ may be given the structure of a (graded) Lie ring. Moreover, if $p>0$ then $L(G)$ has the structure of a restricted Lie algebra over $F_{p}$ (for the basic properties of restricted Lie algebras and their enveloping algebras, see [2, Chapter $5, \S 7])$. In order to obtain a Lie algebra over $F$, set $L:=L(G) \otimes F$.

Received by the editors October 15, 1991.

1980 Mathematics Subject Classification (1985 Revision). Primary 20C05, 20C07; Secondary $17 \mathrm{~B} 60$. 
Quillen's result relates $\operatorname{gr}(F G)$ with the Lie algebra $L$ in the following way. Quillen's Theorem. $\operatorname{gr}(F G) \cong \mathrm{U}(L)$.

Here $\mathrm{U}(L)$ denotes the (universal) enveloping algebra of $L$. It is understood that if $p>0$ then $\mathrm{U}(L)$ stands for the enveloping algebra of $L$ as a restricted Lie algebra.

Our goal here is to prove a certain modification of this result, dealing with arbitrary permutation modules. This approach is motivated by Alperin's recent extension of Jenning's theory to permutation modules for $p$-groups in characteristic $p[1]$ (see also [8]).

So let $G$ be a group acting transitively on a set $\Omega$, and consider the permutation module $M:=F \Omega$ as a left $F G$-module. Define

$$
\operatorname{gr}(M):=\bigoplus_{n \geq 0} \Delta^{n} M / \Delta^{n+1} M .
$$

Let $H \leq G$ be a stabilizer of a point in $\Omega$. The subgroup $H$ gives rise to a Lie subring $K(H)$ of $L(G)$, defined by

$$
K(H):=\bigoplus_{n \geq 1}\left(H \cap D_{n}\right) D_{n+1} / D_{n+1} .
$$

Set $K:=K(H) \otimes F$. Then $K$ is a graded (restricted, if $p>0$ ) Lie subalgebra of $L$. By identifying $L$ with its image in $\mathrm{U}(L)$, we may consider $K$ as a Lie subalgebra of $\mathrm{U}(L)$ and form the (associative) left ideal $\mathrm{U}(L) K$ which it generates in $\mathrm{U}(L)$.

We can now state our main result.

Theorem. With the above notation, $\operatorname{gr}(M) \cong \mathrm{U}(L) / \mathrm{U}(L) K$.

This isomorphism may be interpreted in two ways. Clearly, $\operatorname{gr}(M)$ is a left $\operatorname{gr}(F G)$-module, while $\mathrm{U}(L) / \mathrm{U}(L) K$ is a left $\mathrm{U}(L)$-module. The assertion is that Quillen's isomorphism between $\operatorname{gr}(F G)$ and $\mathrm{U}(L)$ induces an isomorphism between these two modules. But there is another way to interpret the result, without relying on Quillen's theorem. As will become clear in the next section, $\operatorname{gr}(M)$ has a natural structure of a left $\mathrm{U}(L)$-module. The theorem then asserts that, as such, $\operatorname{gr}(M)$ is isomorphic to $\mathrm{U}(L) / \mathrm{U}(L) K$. Note that, in this interpretation, Quillen's theorem is obtained as a corollary (consider the regular representation, in which case $M=F G$ and $K=0$ ).

Now, $L$ and $K$ are graded Lie algebras. As such, they induce a natural grading on $\mathrm{U}(L)$ and on $\mathrm{U}(L) / \mathrm{U}(L) K$. The isomorphism between $\operatorname{gr}(M)$ and $\mathrm{U}(L) / \mathrm{U}(L) K$ respects the corresponding gradings. Applying the PoincaréBirkhoff-Witt theorem, it is therefore possible to compute the Hilbert function associated with $\operatorname{gr}(M)$ as follows.

Corollary. Let

$$
c_{n}=\operatorname{dim}\left(\Delta^{n} M / \Delta^{n+1} M\right),
$$

and let $d_{m}$ be the rank of

$$
\left(H \cap D_{m}\right) D_{m+1} / D_{m+1} \cong H D_{m} / H D_{m+1}
$$

as an abelian group. 
(1) Suppose $p=0$. Then

$$
\sum_{n \geq 0} c_{n} \lambda^{n}=\prod_{m \geq 1}\left(1-\lambda^{m}\right)^{-d_{m}}
$$

(2) Suppose $p>0$. Then

$$
\sum_{n \geq 0} c_{n} \lambda^{n}=\prod_{m \geq 1}\left(\left(1-\lambda^{p m}\right) /\left(1-\lambda^{m}\right)\right)^{d_{m}} .
$$

These formulae extend Jennings's formulae for the dimensions of the homogeneous components of $\operatorname{gr}(F G)$. While the zero characteristic case seems to be new, part (2) of the corollary follows from Alperin's work [1, Theorem 2].

Our theorem, whose proof for the case of finite $p$-groups $(p>0)$ is particularly simple, may be used to derive Alperin's results.

\section{Proof of THE THEOREM}

Some of the arguments applied in the proof of the theorem follow those used by Quillen and Alperin.

Obviously, we may identify $\Omega$ with the set $G / H$ and $M$ with $F[G / H]$. Let $\Delta(H)$ denote the augmentation ideal of $F H$, and set $I:=F G \Delta(H)$. Then $I$ is a left ideal of $M \cong F G / I$. The ideal $I$ gives rise to a graded left ideal in $\operatorname{gr}(F G)$, defined by

$$
\operatorname{gr}(I):=\bigoplus\left(\left(I \cap \Delta^{n}\right)+\Delta^{n+1}\right) / \Delta^{n+1} .
$$

Clearly, $\operatorname{gr}(M) \cong \operatorname{gr}(F G) / \operatorname{gr}(I)$.

Consider the $F$-linear map $f: L \rightarrow \operatorname{gr}(F G)$ given by

$$
f\left(x D_{n+1}\right):=x-1+\Delta^{n+1}, \quad x \in D_{n} .
$$

This map plays an important role in Quillen's proof. It is easy to see that $f$ is well defined and that it is a (restricted, if $p>0$ ) Lie-homomorphism [6]. Hence, $f$ gives rise to a homomorphism of associative algebras $\mathrm{U}(f): \mathrm{U}(L) \rightarrow$ $\operatorname{gr}(F G)$. The map $\mathrm{U}(f)$ induces on $\operatorname{gr}(M)$ (as well as on any other $\operatorname{gr}(F G)$ module) a structure of a $\mathrm{U}(L)$-module. It is clear that $\mathrm{U}(f)$ is surjective, as $f(L)$ contains $\Delta / \Delta^{2}$, which generates $\operatorname{gr}(F G)$ (in fact, by Quillen's theorem, $\mathrm{U}(f)$ is an isomorphism, but we will not make use of this fact).

Observe that, if $h \in H \cap D_{n}$, then $f\left(h D_{n+1}\right)=h-1+\Delta^{n+1} \in \operatorname{gr}(I)$. This shows that $f(K) \subseteq \operatorname{gr}(I)$, and hence $\mathrm{U}(f)(\mathrm{U}(L) K) \subseteq \operatorname{gr}(I)$ as well. We conclude that the composition of $\mathrm{U}(f)$ with the canonical projection $\operatorname{gr}(F G) \rightarrow \operatorname{gr}(F G) / \operatorname{gr}(I) \cong \operatorname{gr}(M)$ gives rise to a well-defined epimorphism $\beta$ : $\mathrm{U}(L) / \mathrm{U}(L) K \rightarrow \operatorname{gr}(M)$.

The proof will be complete if we show that $\beta$ is injective. This is the place where Jennings's theory has to be applied. It is well known that Jennings's construction of a basis for $\operatorname{gr}(F G)$ (see $[3,4]$ ) is valid for arbitrary groups.

Fix a positive integer $n$, and consider the abelian group $L_{n}:=D_{n} / D_{n+1}$ and its subgroup $K_{n}:=\left(H \cap D_{n}\right) D_{n+1} / D_{n+1}$. Choose a basis $\left\{h_{n j}\right\}$ for $K_{n}$ and complete it to a basis of $L_{n}$ by adding the elements $\left\{g_{n i}\right\}$. Choose a fixed linear ordering on $\left\{h_{m j}, g_{n i}\right\}$ in such a way that $g_{n i}<h_{m j}$ for all $n, i, m, j$.

Applying the Poincaré-Birkhoff-Witt theorem for $\mathrm{U}(L)$, we see that the ordered monomials in the basis elements $g_{n i}$ (with exponents $<p$ in each $g_{n i}$ if 
$p>0$ ) form a basis for $\mathrm{U}(L) / \mathrm{U}(L) K$. If $X=\prod\left(g_{n i}\right)^{\alpha_{n i}}$ is such a monomial, we define $\operatorname{deg}(X)=\sum n \alpha_{n i}$.

Now suppose, by contradiction, that $\beta$ is not injective, and let $u=\sum a_{s} X_{s} \in$ $\operatorname{Ker}(\beta)$ be a nonzero element, where $a_{S} \in F$ and $X_{s}=\prod\left(g_{n i}\right)^{\alpha_{s n i}}$ are distinct ordered monomials. Since $\varphi$ respects grading, we may assume that $u$ is homogeneous. This means that, for some $d, \operatorname{deg}\left(X_{s}\right)=d$ for all $s$. By abuse of notation we may consider each monomial $X_{s}$ as an element of $\mathrm{U}(L)$ and identify each $g_{n i}, h_{m j}$ with suitable representatives in $G$ and $H$, respectively. Note that, since $\beta(u)=0$, we obtain $\sum a_{s} \mathrm{U}(f)\left(X_{s}\right) \in \operatorname{gr}(I)$. Clearly, $\mathrm{U}(f)\left(X_{s}\right)=\prod\left(g_{n i}-1\right)^{\alpha_{s n i}}+\Delta^{d+1}$. It follows that

$$
v:=\sum a_{s} \prod\left(g_{n i}-1\right)^{\alpha_{s n i}} \in\left(I \cap \Delta^{d}\right)+\Delta^{d+1} \subseteq F G \Delta(H)+\Delta^{d+1} .
$$

This enables us, using Jennings's method, to express $v$ modulo $\Delta^{d+1}$ as a linear combination of ordered monomials in $\left(g_{n i}-1\right),\left(h_{m j}-1\right)$ (with exponents $<p$ if $p>0)$, all of which terminate with some $\left(h_{m j}-1\right)$. The linear ordering we choose on the set $\left\{\left(g_{n i}-1\right),\left(h_{m j}-1\right)\right\}$ is the one induced from the previous ordering on $\left\{g_{n i}, h_{m j}\right\}$. As noted by Alperin [1], Jennings's method is applicable relative to any given ordering.

Now, to each monomial we associate its (weighted) degree in the usual way (so that $g_{n i}-1$ and $h_{n j}-1$ have degree $n$ ). Jennings's theory tells us that ordered monomials of degree $\leqslant d$ in $\left(g_{n i}-1\right),\left(h_{m j}-1\right)$ form a basis for $F G / \Delta^{d+1}$. In particular, they are linearly independent modulo $\Delta^{d+1}$. Recall that $v$, which is spanned (modulo $\Delta^{d+1}$ ) by monomials of degree $\leqslant d$ all involving some $\left(h_{m j}-1\right)$, can also be expressed as a linear combination $\sum a_{s} \Pi\left(g_{n i}-1\right)^{\alpha_{s n i}}$ of monomials of degree $d$ not involving any $\left(h_{m j}-1\right)$. We conclude that $a_{s}=0$ for all $s$, hence $u=0$. This contradiction completes the proof of the theorem.

Finally we note that, in the case where $p>0$ and $G$ is a finite $p$-group, a particularly simple proof that avoids the use of Jennings's theory is available.

Recall that we have defined an epimorphism $\beta: \mathrm{U}(L) / \mathrm{U}(L) K \rightarrow \operatorname{gr}(M)$, and had to show that it is an isomorphism. Calculating dimensions (using the Poincaré-Birkhoff-Witt theorem), we obtain

$$
\begin{aligned}
\operatorname{dim} \mathrm{U}(L) / \mathrm{U}(L) K & =p^{\operatorname{dim} L-\operatorname{dim} K}=p^{\operatorname{dim} L} / p^{\operatorname{dim} K} \\
& =|G| /|H|=|G / H|=\operatorname{dim} \operatorname{gr}(M) .
\end{aligned}
$$

The conclusion follows.

\section{REFERENCES}

1. J. L. Alperin, Loewy structure of permutation modules for p-groups, Quart. J. Math. Oxford Ser. (2) 39 (1988), 129-133.

2. N. Jacobson, Lie algebras, Wiley-Interscience, New York, 1962.

3. S. A. Jennings, The structure of the group ring of a p-group over a modular field, Trans. Amer. Math. Soc. 50 (1941), 175-185.

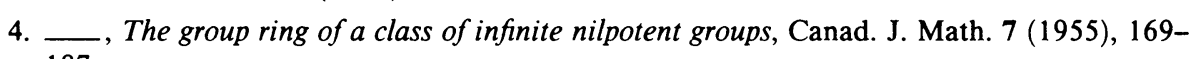
187.

5. M. Lazard, Sur les groupes nilpotents et les anneaux de Lie, Ann. Sci. École Norm. Sup. (4) 71 (1954), 101-190.

6. D. G. Quillen, On the associated graded ring of a group ring, J. Algebra 10 (1968), 411-418. 
7. I. B. S. Passi, Group rings and their augmentation ideals, Lecture Notes in Math., vol. 715, Springer, Berlin, 1979.

8. A. Shalev, Uniserial permutation modules, Quart. J. Math. Oxford Ser. (2) 42 (1991), 375378.

Mathematical Institute, University of OXford, 24-29 St Giles, OXford OX1 3LB, UNITED KINGDOM

Current address: Department of Mathematics, Hebrew University of Jerusalem, Jerusalem 91904, Israel 